\title{
Evaluation of differential peptide loading on tandem mass tag-based proteomic and phosphoproteomic data quality
}

\section{James A Sanford}

Pacific Northwest National Laboratory https://orcid.org/0000-0001-7901-5579

\section{Yang Wang}

Pacific Northwest National Laboratory

Joshua R Hansen

Pacific Northwest National Laboratory

\section{Marina A Gritsenko}

Pacific Northwest National Laboratory

\section{Karl K Weitz}

Pacific Northwest National Laboratory

\section{Cristina E Tognon}

Oregon Health \& Science University

\section{Vladislav A Petyuk}

Pacific Northwest National Laboratory

\section{Wei-Jun Qian}

Pacific Northwest National Laboratory

\section{Tao Liu}

Pacific Northwest National Laboratory

\section{Brian J Druker}

Oregon Health \& Science University

\section{Karin D Rodland}

Pacific Northwest National Laboratory

Paul D. Piehowski ( $\square$ paul.piehowski@pnnl.gov )

Pacific Northwest National Laboratory https://orcid.org/0000-0001-5108-2227

\section{Research}

Keywords: TMT, isobaric labeling, clinical proteomics, phosphoproteomics, Acute Myeloid Leukemia, differential loading

Posted Date: December 29th, 2020 
DOI: https://doi.org/10.21203/rs.3.rs-51123/v2

License: (c) (1) This work is licensed under a Creative Commons Attribution 4.0 International License. Read Full License

Version of Record: A version of this preprint was published at Journal of the American Society for Mass Spectrometry on November 23rd, 2021. See the published version at https://doi.org/10.1021/jasms.1c00169. 


\section{Abstract}

Background: Global and phosphoproteome profiling has demonstrated great utility for the analysis of clinical specimens. One major barrier to the broad clinical application of proteomic profiling is the large amount of biological material required, particularly for phosphoproteomics-currently on the order of 25 mg wet tissue weight, depending on tissue type. For hematopoietic cancers such as acute myeloid leukemia (AML), the sample requirement is in excess of 10 million (1E7) peripheral blood mononuclear cells (PBMCs). Throughout the course of a prospective study, this requirement will certainly exceed what is obtainable from many of the individual patients/timepoints. For this reason, we were interested in examining the impact of differential peptide loading across multiplex channels on proteomic data quality.

Methods: To achieve this, we tested a range of channel loading amounts $(20,40,100,200$, and $400 \mu \mathrm{g}$ of tryptic peptides, or approximately the material obtainable from 5E5, 1E6, 2.5E6, 5E6, and 1E7 AML patient cells) to assess proteome coverage, quantification precision, and peptide/phosphopeptide detection in experiments utilizing isobaric tandem mass tag (TMT) labeling.

Results: As expected, we found that fewer missing values are observed in TMT channels with higher peptide loading amounts compared to those with lower loading. Moreover, channels with lower loading amounts have greater quantitative variability than channels with higher loading amounts. Statistical analysis of the differences in means among the five loading groups showed that the $20 \mu \mathrm{g}$ loading group was significantly different from the $400 \mu \mathrm{g}$ loading group. However, no significant differences were detected among the 40, 100, 200 and $400 \mu$ g loading groups.

Conclusions: These assessment data demonstrate the practical limits of loading differential quantities of peptides across channels in TMT multiplexes, and provide a basis for designing the optimal clinical proteomics study when specimen quantities are limited.

\section{Background}

Mass spectrometry-based proteomic profiling has become a powerful tool for broad quantification of proteins and their post-translational modifications in cancer research (1-6). Due to extensive efforts in the field to benchmark and standardize complex workflows, particularly those of the Clinical Proteomics Tumor Analysis Consortium (CPTAC), proteomics is now being more broadly utilized for clinical research $(7,8)$. Reliable and reproducible quantification of $>10,000$ proteins and $>30,000$ phosphosites is now routinely attainable from numerous mammalian tissue types (7). Integration of deep-scale proteomic analysis of human tumors with genomic data has been shown to improve specificity for identifying pathway alterations caused by tumor associated mutations. Furthermore, phosphoproteome measurements provide information on pathway activation not discernible from genetic measurements and thus offers unique insights into potential therapeutic targets (9-14).

A substantial challenge in the field of clinical proteomics is obtaining the quantity of protein necessary for deep coverage of the proteome, as clinical specimens are often limited in size and available material. 
This challenge is particularly acute in the case of phosphoproteomics, which requires 100 -fold more starting material than global profiling, due to the lower number of peptides with potential phosphosites, and the low percentage of phosphorylation on those sites. The most widely utilized workflow for clinical proteomics studies seeking to achieve deep quantitative proteomic and phosphoproteomic measurements employs a tandem mass tag (TMT) isobaric labeling approach and employs an enrichment step to increase phosphopeptide specificity (15-17). In settings where the deepest achievable coverage of the phosphoproteome is a priority, it is recommended to use on the order of $400 \mathrm{mg}$ of peptides in each TMT channel. While high quality data and coverage of the global proteome can be achieved with significantly less material, phosphoproteome coverage is negatively impacted or requires specialized methods to recover $(18,19)$. This creates a key challenge relating to variability in the amount of protein available per patient, due to external variables in the study that impact sample size availability. In these cases, the investigators need to decide whether to exclude sample-limited patients, reduce the protein loading per patient for the study, or to include that individual patient at reduced protein loading.

In a data-dependent LC-MS/MS experiment, peptide identification is achieved by fragmenting tryptic peptides followed by database searching to obtain sequence information, that is then matched to the parent protein. To obtain confident peptide identifications, a sufficient number of peptide ions are needed to generate high quality MS/MS spectra. A notable advantage of the TMT approach is that many patient samples can be combined to increase the available peptide amount, allowing identification of low abundance species, particularly when coupled with 2-dimensional LC separation (20). Recently, a number of groups have demonstrated a modified TMT labeling scheme that utilizes a "boosting" or "carrier" channel that increases the sensitivity for sample limited samples (21-24). In these approaches, one or more TMT channels are used to label a larger representative sample that is then mixed with the patient samples for analysis. The high abundance of peptides in the boosting channel triggers MS/MS selection and provides the ion flux necessary for quality MS/MS spectra and confident peptide identification, while the reporter ions provide quantitative information on the samples of interest. While this approach enables profound gains in sensitivity for both global (23) and phosphoproteomics (24) workflows, the increased dynamic range of peptide concentration induced by the boosting sample(s) results in compromised quantification with issues such as reduced measurement precision and an increase in missing values $(24,25)$. Further complicating the quantitative precision of TMT experiments is the concept of compositional data-as mass spectrometry measurements are made on a constrained number of ions allowed into the instrument, increasing the proportion of one component will impact the observable amount from other components(26). This issue will be exacerbated in settings where there are significant differences in the quantity of peptides loaded per channel; however the boundaries for acceptable variability between samples remain unclear.

In this study, we set out to interrogate two common challenges of clinical proteomics experiments: the amount of protein available from patient samples of various sizes, and the impact of differential channel loading on global and phosphoproteomics results using a well-established clinical workflow. The overarching goal of this study is to experimentally determine boundary conditions for patient inclusion in clinical proteomics investigations. First, we determined protein yields from patient samples of varying cell 
count. We then used these results to guide an experimental design using 2 TMT 11-plexes to explore a range of peptide loadings that we expect to encounter in executing a clinical proteomics experiment. The results were examined for protein/phosphosite coverage and various aspects of quantitative reproducibility. Our results highlight the protein yields achievable from representative AML samples and demonstrate a thorough examination of the impacts of differential channel loading to provide researchers with a resource to make informed decisions concerning their study design.

\section{Methods}

\section{Cell Counting}

The clinical specimen used for this study was collected with informed consent from the patient according to a protocol approved by the Oregon Health \& Science University institutional review board (IRB 4422; NCT01728402). Three cell pellets, each containing approximately 20 million peripheral blood mononuclear cells (PBMCs) isolated from a single de-identified patient, were combined into a single sterile $1.5 \mathrm{~mL}$ microcentrifuge tube. Each pellet was transferred to the sterile tube with $250 \mathrm{~mL}$ of $1 \mathrm{x}$ phosphate buffered saline (PBS). The original pellet tubes were rinsed with an additional $250 \mathrm{~mL} 1 \mathrm{x}$ PBS that was quantitatively transferred to the pooled sample tube for a final volume of $500 \mathrm{~mL}$. A 20x dilution stock was created to determine cell concentration and live cell count utilizing an Invitrogen Countess II FL cell counter and Invitrogen Countess disposable slides. The dilute cells were combined 1:1 with Trypan blue and $20 \mathrm{~mL}$ was loaded on the slide and read four times; the values were then averaged. Once the total cell concentration was determined, a concentration series was created with three replicates each of 1E7, 5E6, 1E6, 5E5, 1E5, 5E4, and 1E4 cells.

\section{Cell Lysis and Protein Extraction}

Fresh lysis buffer was prepared, containing $8 \mathrm{M}$ urea (Sigma-Aldrich), $50 \mathrm{mM}$ Tris pH 8.0, $75 \mathrm{mM}$ sodium chloride, $1 \mathrm{mM}$ ethylenediamine tetra-acetic acid, $2 \mathrm{mg} / \mathrm{mL}$ Aprotinin (Sigma-Aldrich), $10 \mathrm{mg} / \mathrm{mL}$ Leupeptin (Roche), $1 \mathrm{mM}$ PMSF in EtOH, $10 \mathrm{mM}$ sodium fluoride, $100 \mathrm{~mL}$ of phosphatase inhibitor cocktail 2 and 3 (Sigma-Aldrich), $20 \mathrm{mM}$ PUGNAc, and $0.01 \mathrm{U} / \mathrm{mL}$ Benzonase,. Lysis buffer was added to samples based on cell concentration: $200 \mathrm{~mL}$ for $1 \mathrm{e} 7$ and $5 \mathrm{e} 6 \mathrm{cells}, 40 \mathrm{~mL}$ for $1 \mathrm{e} 6$ cells, $20 \mathrm{~mL}$ for $5 \mathrm{e} 5$ cells, $4 \mathrm{~mL}$ for $1 \mathrm{e} 5$ cells, and $1 \mathrm{~mL}$ for $5 \mathrm{e} 4$ and $1 \mathrm{e} 4$ cells. Once the lysis buffer was added, the samples were vortexed for 10 seconds and then placed in thermomixer for 15 minutes at $4^{\circ} \mathrm{C}$ and $800 \mathrm{RPM}$. To ensure cell lysis, samples were vortexed for an additional 10 seconds and incubated again for 15 minutes utilizing the same settings. After incubation the samples were centrifuged for 10 minutes at $4^{\circ} \mathrm{C}$ and $18000 \mathrm{rcf}$ to remove cell debris. Due to the viscous nature of the samples, additional lysis buffer to double initial volume was added to all samples. Samples were then incubated again twice for 15 minutes at $25^{\circ} \mathrm{C}$ and $500 \mathrm{RPM}$, vortexing samples between incubations then centrifuging to remove cell debris. A single $5 x$ dilution BCA (ThermoFisher) was performed on the supernatant to determine the protein yields for the varying cell concentrations. After examining the results of the protein yields, all the samples were mixed into a large protein pool and concentrated with a 10k spin filter for use in downstream sample prep 
and MS analysis. A final 10x dilution BCA (ThermoFisher) was performed on the supernatant to determine final protein yield for digestion.

\section{Protein Digest}

The pooled protein sample was diluted to a concentration of $8 \mathrm{mg} / \mathrm{mL}$ total protein with $50 \mathrm{mM}$ Tris, $\mathrm{pH}$ 8.0 before reducing the sample with $5 \mathrm{mM}$ dithiothreitol (DTT) (Sigma-Aldrich) for 1 hour in a thermomixer set to $37^{\circ} \mathrm{C}$ and $800 \mathrm{rpm}$. Reduced cystine residues were alkylated with $10 \mathrm{mM}$ iodacetamide (IAA) (Sigma-Aldrich) for 45 minutes in a thermomixer set to $25^{\circ} \mathrm{C}$ and $800 \mathrm{rpm}$ in the dark. The sample was diluted five-fold with $50 \mathrm{mM}$ Tris- $\mathrm{HCl}, \mathrm{pH}=8.0$ and then initially digested with Lys-C (Wako) at a 1:50 enzyme:substrate ratio for 2 hours in a thermomixer set to $25^{\circ} \mathrm{C}$ and $800 \mathrm{rpm}$. Following the initial digest, trypsin (Promega) was added at a 1:50 enzyme:substrate ratio, followed by a 14-hour incubation in a thermomixer set to $25^{\circ} \mathrm{C}$ and $800 \mathrm{rpm}$. The digestion was quenched by acidifying the solution to $1 \%$ formic acid (FA), and the sample was centrifuged for 15 minutes at $1500 \mathrm{rcf}$ to remove any remaining cell debris. Peptides were desalted using a C18 solid phase extraction (SPE) cartridge (Waters Sep-Pak).

\section{TMT Labeling}

The concentration of the pooled peptides was determined by BCA assay, and peptides were aliquoted in discrete amounts, with four replicates each of $20 \mathrm{mg}, 40 \mathrm{mg}, 100 \mathrm{mg}, 200 \mathrm{mg}$, and six replicates of 400 $\mathrm{mg}$ for a total of $3.84 \mathrm{mg}$ for TMT labeling (Thermo Fisher). The samples were then divided evenly into two TMT-11 plex sets. After drying the peptides down in a speed-vac, each sample was reconstituted with $50 \mathrm{mM}$ HEPES, $\mathrm{pH} 8.5$ to a concentration of $5 \mathrm{mg} / \mathrm{mL}$. Each isobaric tag aliquot was dissolved in $40 \mathrm{~mL}$ anhydrous acetonitrile to a final concentration of $20 \mathrm{mg} / \mathrm{mL}$. The tag was added to the sample at a 1:1 $\mu \mathrm{g} / \mu \mathrm{g}$ peptide:label ratio (27) and incubated in a thermomixer for 1 hour at $25^{\circ} \mathrm{C}$ and $400 \mathrm{rpm}$, then diluted to $2.5 \mathrm{mg} / \mathrm{mL}$ with $50 \mathrm{mM}$ HEPES pH 8.5, 20\% acetonitrile (ACN). Finally, the reaction was quenched with $5 \%$ hydroxylamine and incubated on the thermomixer for 15 minutes at $25^{\circ} \mathrm{C}$ and 400 $\mathrm{rpm}$. The samples for each multiplex set were then combined and concentrated in a speed-vac before a final C18 SPE cleanup. Each 11-plex experiment was fractionated into 96 fractions by high pH reversed phase separation using a $3.5 \mathrm{~mm}$ Agilent Zorbax 300 Extend-C18 column (4.6 mm ID x $250 \mathrm{~mm}$ length). Peptides were loaded onto the column in buffer $\mathrm{A}(4.5 \mathrm{mM}$ ammonium formate $(\mathrm{pH} 10)$ in $2 \%(\mathrm{vol} / \mathrm{vol})$ acetonitrile) and eluted off the column using a gradient of buffer $\mathrm{B}(4.5 \mathrm{mM}$ ammonium formate $(\mathrm{pH} 10)$ in $90 \%$ ( $\mathrm{vol} / \mathrm{vol})$ acetonitrile), described in more detail below, for 96 minutes at a flow rate of $1 \mathrm{~mL} / \mathrm{min}$. After fractionation, samples were concatenated into 12 fractions (28). 


\begin{tabular}{|l|l|}
\hline Time interval (minutes) & $\begin{array}{l}\text { Gradient } \\
\text { (\% mobile phase B) }\end{array}$ \\
\hline 0 & 0 \\
\hline 7 & 0 \\
\hline 13 & 16 \\
\hline 73 & 40 \\
\hline 77 & 44 \\
\hline 82 & 60 \\
\hline 96 & 60 \\
\hline
\end{tabular}

\section{Phosphopeptide enrichment using IMAC}

A small aliquot ( $5 \%$ volume) of each of the 12 fractions from both multiplex sets was removed and vialed at $0.1 \mathrm{mg} / \mathrm{mL}$ in $3 \% \mathrm{ACN}, 0.1 \%$ FA for MS analysis of global protein abundance. For phosphopeptide enrichment, the remaining $95 \%$ of the 12 fractions were further combined to create six fractions per plex and dried by speed-vac. Fe $^{3+}$-NTA-agarose beads were freshly prepared for phosphopeptide enrichment using Ni-NTA-agarose beads (Qiagen). Sample peptides were reconstituted to a $0.5 \mathrm{mg} / \mathrm{mL}$ concentration with $80 \% \mathrm{ACN}, 0.1 \%$ TFA and incubated with $40 \mathrm{~mL}$ of the bead suspension for 30 minutes at RT in a thermomixer set at $800 \mathrm{rpm}$. After incubation the beads were washed with $100 \mathrm{~mL} 80 \%$ ACN, $0.1 \%$ TFA and $50 \mathrm{~mL}$ 1\% FA to remove any non-specific binding. Phosphopeptides were eluted off beads with 210 $\mathrm{mL} 500 \mathrm{mM} \mathrm{K}_{2} \mathrm{HPO}_{4}, \mathrm{pH} 7.0$ directly onto C18 stage tips and eluted from C18 material with $60 \mathrm{~mL} \mathrm{50 \%}$ ACN, $0.1 \%$ FA. Samples were dried in speed-vac concentrator and reconstituted with $12 \mathrm{~mL} 3 \%$ ACN, $0.1 \%$ FA prior to MS analysis.

\section{LC-MS/MS analysis}

The pooled proteomics fractions were separated using a Waters nano-Aquity UPLC system (Waters) equipped with a homemade $75 \mu \mathrm{m}$ I.D. $\times 50 \mathrm{~cm}$ length C18 PicoFrit (New Objective) column packed with ReproSil-Pur $120 \AA$, C18-AQ, $1.9 \mu \mathrm{m}$. A 110-min gradient of $100 \%$ mobile phase A $(0.1 \%(\mathrm{v} / \mathrm{v})$ formic acid in water) to $60 \%(\mathrm{v} / \mathrm{v})$ mobile phase $B(0.1 \%(\mathrm{v} / \mathrm{v})$ FA in acetonitrile) was applied to each fraction. The column was equipped with a $20 \mathrm{~cm}$ Nanospray column heater (Phoenix S \& T). The separation was coupled to an Orbitrap Fusion Lumos mass spectrometer (Thermo Fisher) for MS/MS analysis. MS Spectra were collected from 350 to $1800 \mathrm{~m} / \mathrm{z}$ at an MS1 resolution setting of 60,000 , with a maximum injection time of $50 \mathrm{~ms}$ and Orbitrap AGC set to 400,000. The top 20 most intense ions were selected with an isolation width of $0.7 \mathrm{~m} / \mathrm{z}$ for higher energy collision dissociation (HCD); +1 charged species were excluded, and the dynamic exclusion window was set at $45 \mathrm{~s}$. MS2 spectra were acquired at a mass resolution of 50,000, with a maximum injection time of 105 ms and Orbitrap AGC set to 100,000.

Phosphoproteomics fractions were separated as described above, with the LC gradient length extended to $200 \mathrm{~min}$ for each fraction. The separation was coupled to the same Lumos mass spectrometer with the same acquisition method.

\section{Proteomics data processing}


The obtained MS/MS spectra were searched by the MS-GF+ tool (29), against the UniProt human database (downloaded in October 2018) for peptide sequence identification. Carbamidomethylation on cysteine residues, and TMT-11 modifications on lysine residues and the $\mathrm{N}$ termini were set as fixed modifications, with oxidation on methionine residues as a dynamic modification. For determination of TMT labeling efficiency, a separate MS-GF+ search was performed with TMT-11 modifications set as a dynamic modification, and the numbers of identified peptides with and without TMT-11 modifications were used to calculate labeling efficiency. For phosphoproteomics, phosphorylation on serine, threonine, and tyrosine residues were set as dynamic modifications. Localization of phosphorylation modifications was performed using the Ascore algorithm (30). A target-decoy approach was used to control false discovery. Criteria for filtering peptide spectrum matches (PSMs) include precursor mass tolerance as 10 ppm and PepQvalue < 0.01. In both datasets, this resulted in a less than 1\% FDR at the unique peptide level. For identified peptides, the TMT reporter ion intensities were extracted by MASIC (31) with the following filtering thresholds: signal-to-noise ratio $=0$; interference score $=0.9$. Data from all fractions of a multiplex (12 fractions for global abundance and 6 fractions for phosphopeptide abundance per plex) were aggregated based on common peptides or proteins (global proteomics) or phosphopeptides (phosphoproteomics). Data aggregation and processing was performed with R Studio software-based tools developed by our team and available via Github (https://github.com/vladpetyuk).

\section{Statistical analysis}

Quantification reproducibility was assessed by calculating the percent coefficient of variation (\%CV) among replicates of each loading group using TMT reporter ion intensities that had been aggregated to the peptide or protein level and global median shift-normalized. For downstream analysis, raw reporter ion intensities were aggregated and log2-transformed, and data for each sample within a TMT set was divided by the universal reference channel of that plex (designated as channel 131C). Within each sample, the central tendency method was used for data normalization for the global data based on medians (32). For phosphoproteomics, each TMT channel was normalized based on the coefficients derived from global data (33). Principal component analysis (PCA) was applied to demonstrate the clustering of the different loading groups. Unequal variance t-tests were performed to determine statistical differences between the individual loading groups. Statistical results were adjusted for multiple hypothesis testing using the Benjamini-Hochberg procedure, and adjusted p-value $<0.05$ was considered as the statistical significance cutoff. Data analysis and statistical tests were implemented in $\mathrm{R}$ language for statistical computing using the following packages: dplyr, reshape2, vp.misc, tibble, tidyverse, and ggplot2.

\section{Results}

\section{Experimental design, protein yield, and peptide identifications}

In clinical proteomic studies, biospecimen availability can be a constraining factor depending on the tissue of interest being analyzed. In this experiment, we used peripheral blood mononuclear cells 
(PBMCs) isolated from AML patients as a model and followed a modified version of the CPTAC TMTbased clinical proteomics pipeline (7). An illustration of the general workflow used to process the samples, from cell lysis through LC-MS/MS analysis, is presented in Figure 1. To determine the amount of protein obtainable from patient samples of varying sizes and volumes, we set up three replicates each of $A M L$ patient cells representing $1 E 7,5 E 6,1 E 6,5 E 5,1 E 5,5 E 4$, and $1 E 4$ total cell counts. Samples were lysed in proportional amounts of lysis buffer; protein concentration was determined by BCA assay and total protein yield was calculated. As shown in Figure 2, achieving the most commonly used amount of $400 \mu \mathrm{g}$ total protein requires greater than 10 million cells (Figure $2 \mathrm{~A}$ ). Lower numbers of cells resulted in a linear decrease in protein yields, with less than $1 \mu \mathrm{g}$ of total protein extracted from smaller amounts of cells (5E4 and $1 \mathrm{E} 4$ cells). While these lower protein yields are substantially less than what is preferred for multiplexed proteomic studies, the sample sizes represent total amounts of biomaterial isolated in realworld clinical settings where available material is constrained. In these cases, researchers may face difficult decisions about inclusion of samples in experimental designs; thus, we set out to evaluate the impact of lower and varying protein amounts on LC-MS/MS peptide and protein identification.

As technical variability in proteomics workflows (i.e. protein extraction and digestion) is unavoidableand likely amplified when sample size, protein amount and buffer volumes differ-we opted to pool all protein extracted from the different cell pellets and carry out digestion in a single reaction (Figure 1). From this homogenous pool of starting material, we generated aliquots containing either $400 \mu \mathrm{g}$ (the conventional amount loaded into each channel of clinical TMT proteomics experiments), $200 \mu \mathrm{g}, 100 \mu \mathrm{g}$, $40 \mu \mathrm{g}$, or $20 \mu \mathrm{g}$ of total peptides to assess the effect of variable peptide amount. We then prepared 2 sets of samples for labeling with TMT 11-plex reagents, with each set containing 2 replicates of each peptide aliquot distributed randomly through the first 10 TMT channels (Figure 2B). An additional aliquot of 400 $\mu \mathrm{g}$ of peptides was included in each plex for use as a universal reference and was assigned to the $131 \mathrm{C}$ channel. This experimental design-standardizing the source of peptides in each channel from a large pool homogenized after digest and clean up-ensures that differences detected in downstream data processing and analysis are based on peptide loading and not sample processing. Following TMT labelling, the samples were mixed into their respective multiplexes, desalted by C18 SPE, fractionated by high $\mathrm{pH}$ reverse-phase HPLC, and concatenated into 12 total fractions. An aliquot of each fraction was removed for global proteomics analysis, and the remaining material was further concatenated into 6 fractions and underwent IMAC phosphopeptide enrichment. Global and phosphopeptide-enriched samples were analyzed by LC-MS/MS, and data was used to evaluate reporter ion intensities for each TMT channel as well as calculate total numbers of peptide and protein identifications.

Figure $2 \mathrm{C}$ displays the overall labeling efficiency calculated in each plex as well as the total reporter ion intensities acquired for each channel across both TMT11 multiplexes. In general, channels with equivalent peptide loadings showed consistent reporter ion intensities within and across the 2 plexes (Figure 2C). Furthermore, the median intensities for different channels increase linearly with the amount of peptide loaded per channel, demonstrating the general quantitative information achievable through utilization of TMT methodology (Figure 2D). Data from global and phospho-enriched fractions were used to determine the number of unique peptides and proteins identified from these samples (Table 1). 
Additionally, Table 1 displays the number of peptides/proteins from global and phosphoproteomic datasets that are quantified in $25 \%, 33 \%, 50 \%$, and $100 \%$ of samples-cut-offs commonly applied to proteomics datasets prior to statistical analysis.

\section{Table 1. Peptide and protein identifications.}

\begin{tabular}{|c|c|c|}
\hline & Global Proteomics & Phosphoproteomics \\
\hline Unique peptide identifications (total) & 138373 & 27351 \\
\hline $\begin{array}{l}\text { Unique peptides } \\
\text { Quantified in }>25 \% \text { of samples }\end{array}$ & $\begin{array}{l}137302 \\
(99.2 \%)\end{array}$ & $\begin{array}{c}26753 \\
(97.8 \%)\end{array}$ \\
\hline $\begin{array}{l}\text { Unique peptides } \\
\text { Quantified in }>33 \% \text { of samples }\end{array}$ & $\begin{array}{l}134399 \\
(97.1 \%)\end{array}$ & $\begin{array}{l}24230 \\
(88.6 \%)\end{array}$ \\
\hline $\begin{array}{l}\text { Unique peptides } \\
\text { Quantified in }>50 \% \text { of samples }\end{array}$ & $\begin{array}{l}118418 \\
(85.6 \%)\end{array}$ & $\begin{array}{l}17409 \\
(63.7 \%)\end{array}$ \\
\hline $\begin{array}{l}\text { Unique peptides } \\
\text { Quantified in } 100 \% \text { of samples }\end{array}$ & $\begin{array}{l}45925 \\
(33.2 \%)\end{array}$ & $\begin{array}{c}3366 \\
(12.3 \%)\end{array}$ \\
\hline Unique protein identifications (total) & 8926 & NA \\
\hline $\begin{array}{l}\text { Unique proteins } \\
\text { Quantified in }>25 \% \text { of samples }\end{array}$ & $\begin{array}{c}8910 \\
(99.8 \%)\end{array}$ & NA \\
\hline $\begin{array}{l}\text { Unique proteins } \\
\text { Quantified in }>33 \% \text { of samples }\end{array}$ & $\begin{array}{c}8887 \\
(99.6 \%)\end{array}$ & $\mathrm{NA}$ \\
\hline $\begin{array}{l}\text { Unique proteins } \\
\text { Quantified in }>50 \% \text { of samples }\end{array}$ & $\begin{array}{c}8722 \\
(97.7 \%)\end{array}$ & NA \\
\hline $\begin{array}{l}\text { Unique proteins } \\
\text { Quantified in } 100 \% \text { of samples }\end{array}$ & $\begin{array}{c}7641 \\
(85.6 \%)\end{array}$ & $\overline{\mathrm{NA}}$ \\
\hline
\end{tabular}

Table 1. Number of unique peptides and proteins identified from global proteomics datasets and phosphoproteomics datasets across the two experimental TMT11 multiplexes. Unique peptide counts were filtered based on presence in $>25 \%, 33 \%, 50 \%$, and $100 \%$ of sample channels, and percentages displayed represent the fraction of overall unique peptide identifications that pass the filtering criteria.

\section{Effects of differential peptide loading on missing data}

While our results demonstrate that reporter ion intensities correlate strongly with peptide loadings and multiplex experiments with differentially loaded channels yield good proteome coverage, a larger question remains regarding the impact of differential loading on quantitative data reproducibility and reliability. In multiplex proteomic experiments, missing data-that is, identified spectra where reporter ion intensities are not detected for one or more TMT channels-pose a significant challenge, especially when comparing across multiple TMT plexes (25). Indeed, when evaluating these datasets, an obvious trend of increasing missingness was evident in the data as peptide loading amounts decreased (Figure 3A). In general, this issue was more pronounced in the phosphoproteomic datasets, as the differences in samples was likely exacerbated by the phosphopeptide enrichment protocol. The effects of missing data in global proteomics datasets can be largely mitigated by rolling peptide identifications/ quantifications up to the protein level (Figure 3A); however, in cases where comparisons are to be made between individual peptide intensities (i.e. phosphoproteomic datasets), missing data can have tremendous implications on downstream statistical analysis. Consistent with the increased levels of missing data as 
peptide loadings decrease, comparison of the peptides identified in all replicates of each loading group illustrates that as channel loading decreases to $40 \mu \mathrm{g}$ or $20 \mu \mathrm{g}$ we begin to see increasing numbers of peptides that are not quantified in these samples (Figure 3B-C).

We sought to compare the levels of missing data in these differentially loaded TMT plexes with those that might arise in a standard TMT experiment where all channels contain equivalent peptide loadings. To this end, we analyzed data generated in our laboratory from an experiment using two TMT11 multiplexes where all channels were loaded with $400 \mu \mathrm{g}$ of peptides derived from similar biological material (human AML cell lines), processed with the same sample preparation protocols, fractionated into 12 global fractions and 6 phospho fractions per plex, and analyzed on the same instrument with the same acquisition settings (this data is deposited on the MassIVE respository under the same accession as the data from the differential loading experiment). As illustrated in Table 2, differential peptide loading results in higher levels of missing data within multiplexes: on average, only $36 \%$ of phosphopeptides were quantified in all 10 channels, and only $83 \%$ of phosphopeptides were quantified in more than 6 channels (Table 2). These rates of missing data are significantly higher than those seen in the standard loading experiment-on average, greater than $95 \%$ of phosphopeptides are observed in all channels, and over $99 \%$ are observed in more than 6 channels (Table 2). While the issue of missing data is more apparent in phosphoproteomics measurements likely due to the low abundance of enriched phosphopeptides, the problem still exists in global proteomics. At the peptide level, only $76 \%$ of observations were quantified in all channels of either multiplex, while $96 \%$ of observations were quantified in more than 6 channels (Table 2). Global proteomics measurements benefit from the aggregation of data to the protein level; when evaluating quantification at the protein level, $96 \%$ of observations have values in all channels of either plex (Table 2). Again, these values are lower than standard, equally loaded TMT experiments, where $\sim 99 \%$ of peptides and proteins are typically observed in all channels (Table 2). In all cases, the higher levels of missing data occur in channels with lower peptide loading, which we attribute to the reduced signal-to-noise ratio for these channels.

Table 2. Missing data in differential loading vs. standard loading TMT experiments. 


\begin{tabular}{|c|c|c|c|c|c|c|}
\hline & \multicolumn{2}{|c|}{$\begin{array}{l}\text { Phosphoproteomics } \\
\text { Phosphopeptide-level }\end{array}$} & \multicolumn{2}{|c|}{$\begin{array}{l}\text { Global Proteomics } \\
\text { Peptide-level }\end{array}$} & \multicolumn{2}{|c|}{$\begin{array}{l}\text { Global Proteomics } \\
\text { Protein-level }\end{array}$} \\
\hline & $\begin{array}{l}\text { Differential } \\
\text { Loading }\end{array}$ & $\begin{array}{l}\text { Standard } \\
\text { Loading }\end{array}$ & $\begin{array}{l}\text { Differential } \\
\text { Loading }\end{array}$ & $\begin{array}{l}\text { Standard } \\
\text { Loading }\end{array}$ & $\begin{array}{l}\text { Differential } \\
\text { Loading }\end{array}$ & $\begin{array}{l}\text { Standard } \\
\text { Loading }\end{array}$ \\
\hline Observations & $\begin{array}{l}\text { Average. } \\
\text { \% Total }\end{array}$ & $\begin{array}{l}\text { Average. } \\
\text { \% Total }\end{array}$ & $\begin{array}{l}\text { Average. } \\
\text { \% Total }\end{array}$ & $\begin{array}{l}\text { Average. } \\
\text { \% Total }\end{array}$ & $\begin{array}{l}\text { Average. } \\
\text { \% Total }\end{array}$ & $\begin{array}{l}\text { Average. } \\
\text { \% Total }\end{array}$ \\
\hline 10 & 35.91 & 95.86 & 76.09 & 98.90 & 95.63 & 99.89 \\
\hline 9 & 55.89 & 97.95 & 88.43 & 99.42 & 97.91 & 99.95 \\
\hline 8 & 70.24 & 98.84 & 92.95 & 99.65 & 98.80 & 99.97 \\
\hline 7 & 82.78 & 99.27 & 96.05 & 99.76 & 99.35 & 99.98 \\
\hline 6 & 93.02 & 99.53 & 98.18 & 99.84 & 99.70 & 99.99 \\
\hline 5 & 97.16 & 99.69 & 99.10 & 99.89 & 99.85 & 99.99 \\
\hline 4 & 98.79 & 99.82 & 99.55 & 99.93 & 99.93 & 99.99 \\
\hline 3 & 99.55 & 99.91 & 99.82 & 99.97 & 99.98 & 99.99 \\
\hline 2 & 99.9 & 99.96 & 99.95 & 99.99 & 99.99 & 99.99 \\
\hline 1 & 100 & 100 & 100 & 100 & 100 & 100 \\
\hline Total IDs & 20,818 & 26,428 & 103,582 & 119,335 & 8,514 & 7,214 \\
\hline
\end{tabular}

Table 2. Phosphoproteomics and global proteomics data from two TMT11 multiplexes from this experiment (Differential Loading) were compared with data acquired across two TMT11 multiplexes of peptides derived from a similar biological source, but with equal loading of 400 $\mu \mathrm{g}$ in all channels (Standard Loading). This table quantifies the percentage of total peptides, proteins, or phosphopeptides identified that were quantified in 1 through all 10 TMT channels (excluding the reference channel).

\section{Statistical differences induced by differential channel loading}

Before making any comparisons across differential loading groups, data for each sample were normalized by the central tendency method based on median values $(32,33)$, a standard approach in proteomics data analysis that accounts for technical variations between samples (Supplemental Figure 1). Following median normalization, principal component analysis (PCA) of both global and phosphoproteomic datasets indicate that peptide loading influences data quantification at a certain threshold: while $400 \mu \mathrm{g}, 200 \mu \mathrm{g}$, and $100 \mu \mathrm{g}$ samples all group reasonably close to one another postnormalization, samples with $40 \mu \mathrm{g}$ or $20 \mu \mathrm{g}$ of peptides drift away from the other samples and show more variation within the replicates (Figure 4A-B). Additionally, the reproducibility within each loading group decreases as a function of peptide quantity, demonstrated by plotting the percent coefficient of variation calculated from raw TMT reporter ion intensities among replicates in both global and phosphoproteomic datasets (Figure 4A-B). These data indicate that as the amount of peptide loaded per channel decreases, precision of reporter ion intensity measurement decreases. In settings where comparisons are to be made between channels (i.e. when comparing patient samples), large variations in amount of material loaded will likely impair the ability to discern statistically relevant biological differences.

While preliminary visualization by principal component analysis (PCA) plots suggest that samples with lower peptide loading cluster less tightly than samples with higher loadings, we sought to gain a better understanding of the effects of differential channel loading on statistical data analysis. Based on the unequal variances detected among the loading groups, we used unequal variance t-tests to compare each 
sample loading group to the $400-\mu \mathrm{g}$ sample group. As samples were all derived from a common pool of peptide digest, we employ the assumption that there should be no statistically significant differences between peptide loading levels. In both global and phosphoproteomic datasets, we observe more differences from the 400- $\mathrm{gg}$ sample group as peptide loading decreases. While few proteins or peptides remain significantly different after correction for multiple hypothesis testing (defined as BenjaminiHochberg adjusted $p$-value $<0.05$ ), p-value histograms when comparing the $20-\mu \mathrm{g}$ samples or $40-\mu \mathrm{g}$ samples with the 400- $\mu$ g sample group show a more anti-conservative distribution suggesting larger quantitative differences (Figure 4C-D). Combined, these data demonstrate that using standard data normalization methods, up to 4-fold differences in channel loading can be effectively corrected and not have significant impacts on quantification precision, while more drastic differences in channel loading (i.e. 10-fold or 20-fold) may cause difficulties when trying to detect differences between patient samples.

\section{Discussion}

In this study, we explored the practical limits for loading differential amounts of peptides across channels in a TMT11 multiplex experiment. Biologically identical material derived from a common peptide pool generated from the white blood cells of a single patient was analyzed in aliquots ranging from the amount most commonly used for clinical proteomics experiments $-400 \mu \mathrm{g}$ of peptide per TMT channeldown to $20 \mu \mathrm{g}$ per channel (representing 20 -fold less peptide). This experimental design allowed for isolation of the effect of peptide loading quantity and eliminated much of the variation that would be introduced during sample processing steps-particularly, related to the processing of samples of varying quantity/ concentration-and this should be taken into consideration when interpreting results. Samples were randomly divided across 2 TMT11 multiplexes and processed for LC-MS/MS-based global proteomics and phosphoproteomics measurements using a standard clinical proteomics protocol developed under the CPTAC. Analysis of the data generated from this work aimed to address two aspects of concern for quantitative proteomics experiments: missing data and data reproducibility.

It is readily clear from our results that peptide quantity and missing data are inversely correlated at the channel level-that is, as lower amounts of peptide are labeled in a channel, the number of features for which no quantitative information is extracted increases. While it is well-established that missing data is a major challenge in proteomics analyses $(34,35)$, this typically only arises when comparing data acquired across multiple TMT sets, and there are generally very low rates of missingness within a single TMT plex when all channels contain equivalent amounts of labeled peptide (36). Our data illustrates an exacerbation of the cross-plex missing data problem, with more than $50 \%$ of phosphopeptide identifications failing to be quantified in channels loaded with only $20 \mu \mathrm{g}$, as well as a significant increase in the levels of missing data within a single plex (Table 2). In addition to higher levels of missing data, TMT channels loaded with less peptide also displayed increased variation among the replicates. Coefficient of variation values steadily increased as peptide loadings were reduced, with noticeable differences present in the $40 \mu \mathrm{g}$ and $20 \mu \mathrm{g}$ samples, particularly in phosphoproteomics data. Additionally, PCA analysis illustrated that samples in the $40 \mu \mathrm{g}$ and $20 \mu \mathrm{g}$ groups began to separate away from the $400 \mu \mathrm{g}, 200 \mu \mathrm{g}$, and $100 \mu \mathrm{g}$ samples, which all clustered tightly together. Statistical 
testing of each different loading group to the $400-\mu \mathrm{g}$ group revealed differences in the $40-\mu \mathrm{g}$ and $20-\mu \mathrm{g}$ groups, and while most statistical significance was corrected by multiple hypothesis testing adjustments, distributions of $p$-values from these comparisons are noticeably weighted towards lower $p$-values indicative of discrepancies in quantitation. Together, this data demonstrates that 10 -fold or greater sample loading differences lead to increased variation and negative effects on TMT measurement precision that will likely impact the confidence in statistical interpretations of the samples.

The impacts of differential peptide loading on missing data and quantitative reproducibility have a compounding effect when one is interested in comparing samples. First, the amount of missing data present in samples with lower loadings reduces the number of observations for which statistical analysis can be confidently performed. Second, among the features that have enough observations for statistical testing, quantitation is negatively impacted in samples with lower peptide loadings-likely due to signals closer to the noise level of the mass spectrometer, leading to increased variation when compared with higher loading groups. Importantly, the samples in this experiment were all derived from a common biological source; in true clinical studies, negative impacts on reproducibility will increase quantitative variability and reduce the statistical power, hindering the ability to confidently detect differences between patients, tissue types, or normal vs. diseased samples $(33,37)$. Furthermore, as true clinical samples are processed individually in the laboratory, differences in protein yields will likely increase the variation among samples and thus decrease the range of loading differences that are compatible with TMT multiplexing.

To Illustrate this point, we calculated and plotted the standard deviations for each phosphopeptide when using only the samples loaded with $400 \mu \mathrm{g}$ of peptide, or when combining any of the other loading groups with the $400 \mu \mathrm{g}$ samples. As lower loading groups are combined with the $400 \mu \mathrm{g}$ samples, standard deviation measurements increase (Supplemental Figure 2A). Using the mean standard deviation for each sample set, we estimated the sample size per group necessary to measure fold changes ranging from 1.2 to 2 -fold with statistical significance (Supplemental Figure 2B). While larger fold changes are detectable in any sample set with a reasonable number of patients per group ( $<2$ patients per group for equal $400 \mu \mathrm{g}$ loadings; 7.8 patients per group with 20 -fold loading differences), smaller fold changes (1.2 and 1.4 fold) require a dramatic increase in the number of patients per group to detect statistical significance when combining the more variable $20 \mu$ loading group with the $400 \mu$ g loading group. Compared to equal $400 \mu \mathrm{g}$ loading, where only 4.8 or 2.4 patients per group are required to detect fold changes of 1.2 and 1.4 , respectively, combining $400 \mu \mathrm{g}$ and $20 \mu \mathrm{g}$ loadings increase the necessary sample sizes to 100 and 29 patients per group. From a clinical standpoint, obtaining and analyzing samples from this number of subjects can be a major challenge.

Reproducible and in-depth proteomic analysis of samples smaller than 500,000 cells requires significant improvements in sensitivity over standard approaches. The necessary technological improvements are actively being pursued in our lab $(24,38-41)$ and others $(23,42-44)$ with great success in recent years. However, much more work is needed to make these technologies available to less specialized laboratories.

Page 14/23 


\section{Conclusions}

From this work, we conclude that when designing TMT multiplex experiments, researchers should aim to keep peptide loadings below 4 -fold differences across channels, to minimize impacts on measurement precision. At this level of variation, it is possible to minimize the effects on downstream statistical analysis with common data normalization techniques such as global median-centering. While we recommend from our analysis to maintain loading differences of 4 -fold or less, researchers can use this data to independently assess the tradeoffs associated with including sample-limited patients in their study design. For example, in cases where only a small number of patients have limited material and $\mathrm{n}$ is large, it may be most prudent to leave out these patients to preserve the highest quality data. Conversely, the use of sample-limited patients may be required to create a properly balanced study design and the researcher needs to account for loss of statistical power resulting from increased variance and data missingness.

\section{Abbreviations}

AML: acute myeloid leukemia

PBMCs: Peripheral blood mononuclear cells

TMT: tandem mass tag

LC-MS/MS: liquid chromatography tandem mass spectrometry

PBS: phosphate buffered saline

DTT: dithiothreitol

IAA: iodacetamide

SPE: solid phase extraction

IMAC: immobilized metal affinity chromatography

CPTAC: Clinical Proteomics Tumor Analysis Consortium

PCA: principal component analysis

\section{Declarations}

Ethics approval and consent to participate: The clinical specimen used for this study was collected with informed consent from the patient according to a protocol approved by the Oregon Health \& Science University institutional review board (IRB 4422; NCT01728402). 
Consent for publication: Not applicable.

Availability of data and material: Mass spectrometry proteomics data have been deposited to the ProteomeXchange Consortium (45) via the MassIVE partner repository (http://massive.ucsd.edu ) with the dataset identifier MSV000086417.

Competing interests: The authors have no conflicts of interest to declare

Funding: This work was supported by The Proteogenomic Translational Research Center for Clinical Proteomics Tumor Analysis Consortium, U01 CA214116, from NIH NCI to BJD and KDR and U24CA210955 from NIH NCI to TL, and R01 DK122160 from NIH NIDDK to WQ.

Authors' contributions: The study design was conceived by PDP, WQ, TL, BJD and KDR. Patient samples were provided by BJD. Patient samples were prepared by CET, JRH, and MAG. Mass spectrometry analysis was carried out by KKW. Data analysis was done by YW, JAS, VAP and PDP. The manuscript was written by JAS and PDP, with critical review from KDR and CET.

Acknowledgements: Parts of this research were performed using EMSL, a national scientific user facility sponsored by the Department of Energy's Office of Biological and Environmental Research and located at PNNL.

\section{References}

1. Doll S, Gnad F, \& Mann M (2019) The Case for Proteomics and Phospho-Proteomics in Personalized Cancer Medicine. PROTEOMICS - Clinical Applications 13(2):1800113.

2. Piehowski PD, et al. (2018) Residual tissue repositories as a resource for population-based cancer proteomic studies. Clinical Proteomics 15(1):26.

3. Bradshaw RA, Hondermarck H, \& Rodriguez H (Cancer Proteomics and the Elusive Diagnostic Biomarkers. PROTEOMICSO(ja):1800445.

4. Jimenez CR, Zhang H, Kinsinger CR, \& Nice EC (2018) The cancer proteomic landscape and the HUPO Cancer Proteome Project. Clinical Proteomics 15(1):4.

5. Shah P, et al. (2015) Integrated Proteomic and Glycoproteomic Analyses of Prostate Cancer Cells Reveal Glycoprotein Alteration in Protein Abundance and Glycosylation. Mol Cell Proteomics 14(10):2753-2763.

6. Zhou Y, et al. (2020) An Integrated Workflow for Global, Glyco-, and Phospho-proteomic Analysis of Tumor Tissues. Anal Chem 92(2):1842-1849.

7. Mertins $\mathrm{P}$, et al. (2018) Reproducible workflow for multiplexed deep-scale proteome and phosphoproteome analysis of tumor tissues by liquid chromatography-mass spectrometry. Nature Protocols 13(7):1632-1661.

8. Mertins $\mathrm{P}$, et al. (2013) Integrated proteomic analysis of post-translational modifications by serial enrichment. Nature Methods 10:634. 
9. Mertins P, et al. (2016) Proteogenomics connects somatic mutations to signalling in breast cancer. Nature 534:55.

10. Zhang B, et al. (2014) Proteogenomic characterization of human colon and rectal cancer. Nature 513:382.

11. Zhang $\mathrm{H}$, et al. (2016) Integrated proteogenomic characterization of human high-grade serous ovarian cancer. Cell 166(3):755-765.

12. Vasaikar S, et al. (2019) Proteogenomic Analysis of Human Colon Cancer Reveals New Therapeutic Opportunities. Cell 177(4):1035-1049 e1019.

13. Clark DJ, et al. (2020) Integrated Proteogenomic Characterization of Clear Cell Renal Cell Carcinoma. Cell 180(1):207.

14. Dou Y, et al. (2020) Proteogenomic Characterization of Endometrial Carcinoma. Cell 180(4):729-748 e726.

15. McAlister GC, et al. (2012) Increasing the multiplexing capacity of TMTs using reporter ion isotopologues with isobaric masses. Anal Chem 84(17):7469-7478.

16. Kreuzer J, Edwards A, \& Haas W (2019) Multiplexed quantitative phosphoproteomics of cell line and tissue samples. Methods Enzymol 626:41-65.

17. Paulo JA, Jedrychowski MP, Chouchani ET, Kazak L, \& Gygi SP (2018) Multiplexed Isobaric TagBased Profiling of Seven Murine Tissues Following In Vivo Nicotine Treatment Using a Minimalistic Proteomics Strategy. Proteomics 18(10):e1700326.

18. Ahrne E, et al. (2015) Exploiting the multiplexing capabilities of tandem mass tags for highthroughput estimation of cellular protein abundances by mass spectrometry. Methods 85:100-107.

19. Satpathy $S$, et al. (2020) Microscaled proteogenomic methods for precision oncology. Nat Commun 11(1):532.

20. High AA, et al. (2017) Deep Proteome Profiling by Isobaric Labeling, Extensive Liquid Chromatography, Mass Spectrometry, and Software-assisted Quantification. J Vis Exp (129).

21. Leoni E, et al. (2019) Combined Tissue-Fluid Proteomics to Unravel Phenotypic Variability in Amyotrophic Lateral Sclerosis. Scientific Reports 9(1):4478.

22. Russell CL, et al. (2017) Combined tissue and fluid proteomics with Tandem Mass Tags to identify low-abundance protein biomarkers of disease in peripheral body fluid: An Alzheimer's Disease case study. Rapid Communications in Mass Spectrometry 31(2):153-159.

23. Budnik B, Levy E, Harmange G, \& Slavov N (2018) SCoPE-MS: mass spectrometry of single mammalian cells quantifies proteome heterogeneity during cell differentiation. Genome Biology 19(1):161.

24. Yi L, et al. (2019) Boosting to Amplify Signal with Isobaric Labeling (BASIL) Strategy for Comprehensive Quantitative Phosphoproteomic Characterization of Small Populations of Cells. Analytical Chemistry 91(9):5794-5801. 
25. Brenes A, Hukelmann J, Bensaddek D, \& Lamond Al (2019) Multibatch TMT Reveals False Positives, Batch Effects and Missing Values. Molecular \& cellular proteomics : MCP 18(10):1967-1980.

26. O'Brien JJ, et al. (2018) Compositional Proteomics: Effects of Spatial Constraints on Protein Quantification Utilizing Isobaric Tags. J Proteome Res 17(1):590-599.

27. Zecha J, et al. (2019) TMT Labeling for the Masses: A Robust and Cost-efficient, In-solution Labeling Approach. Molecular \&amp; Cellular Proteomics 18(7):1468-1478.

28. Wang Y, et al. (2011) Reversed-phase chromatography with multiple fraction concatenation strategy for proteome profiling of human MCF10A cells. Proteomics 11(10):2019-2026.

29. Kim S \& Pevzner PA (2014) MS-GF+ makes progress towards a universal database search tool for proteomics. Nat Commun 5:5277.

30. Beausoleil SA, Villen J, Gerber SA, Rush J, \& Gygi SP (2006) A probability-based approach for highthroughput protein phosphorylation analysis and site localization. Nat Biotechnol 24(10):1285-1292.

31. Monroe ME, Shaw JL, Daly DS, Adkins JN, \& Smith RD (2008) MASIC: A software program for fast quantitation and flexible visualization of chromatographic profiles from detected LC-MS(/MS) features. Computational Biology and Chemistry 32(3):215-217.

32. Callister SJ, et al. (2006) Normalization approaches for removing systematic biases associated with mass spectrometry and label-free proteomics. J Proteome Res 5(2):277-286.

33. Piehowski PD, et al. (2013) Sources of technical variability in quantitative LC-MS proteomics: human brain tissue sample analysis. Journal of proteome research 12(5):2128-2137.

34. Webb-Robertson B-JM, et al. (2015) Review, Evaluation, and Discussion of the Challenges of Missing Value Imputation for Mass Spectrometry-Based Label-Free Global Proteomics. Journal of Proteome Research 14(5):1993-2001.

35. Lazar C, Gatto L, Ferro M, Bruley C, \& Burger T (2016) Accounting for the Multiple Natures of Missing Values in Label-Free Quantitative Proteomics Data Sets to Compare Imputation Strategies. Journal of Proteome Research 15(4):1116-1125.

36. Chen LS, Wang J, Wang X, \& Wang P (2017) A MIXED-EFFECTS MODEL FOR INCOMPLETE DATA FROM LABELING-BASED QUANTITATIVE PROTEOMICS EXPERIMENTS. Ann Appl Stat11(1):114138.

37. Levin Y (2011) The role of statistical power analysis in quantitative proteomics. PROTEOMICS 11(12):2565-2567.

38. Zhu Y, et al. (2018) Nanodroplet processing platform for deep and quantitative proteome profiling of 10-100 mammalian cells. Nature Communications 9(1):882.

39. Piehowski PD, et al. (2020) Automated mass spectrometry imaging of over 2000 proteins from tissue sections at 100- $\mu \mathrm{m}$ spatial resolution. Nature Communications 11(1):8.

40. Dou M, et al. (2019) High-Throughput Single Cell Proteomics Enabled by Multiplex Isobaric Labeling in a Nanodroplet Sample Preparation Platform. Analytical Chemistry 91(20):13119-13127. 
41. Dou M, et al. (2019) Automated Nanoflow Two-Dimensional Reversed-Phase Liquid Chromatography System Enables In-Depth Proteome and Phosphoproteome Profiling of Nanoscale Samples. Analytical Chemistry 91(15):9707-9715.

42. Lombard-Banek C, Moody SA, \& Nemes P (2016) Single-Cell Mass Spectrometry for Discovery Proteomics: Quantifying Translational Cell Heterogeneity in the 16-Cell Frog (Xenopus) Embryo. Angewandte Chemie International Edition 55(7):2454-2458.

43. Myers SA, et al. (2019) Streamlined Protocol for Deep Proteomic Profiling of FAC-sorted Cells and Its Application to Freshly Isolated Murine Immune Cells. Molecular \&amp; Cellular Proteomics 18(5):995-1009.

44. Wu R, Pai A, Liu L, Xing S, \& Lu Y (2020) NanoTPOT: Enhanced Sample Preparation for Quantitative Nanoproteomic Analysis. Analytical Chemistry 92(9):6235-6240.

45. Deutsch EW, et al. (2020) The ProteomeXchange consortium in 2020: enabling 'big data' approaches in proteomics. Nucleic Acids Res 48(D1):D1145-D1152.

\section{Figures}

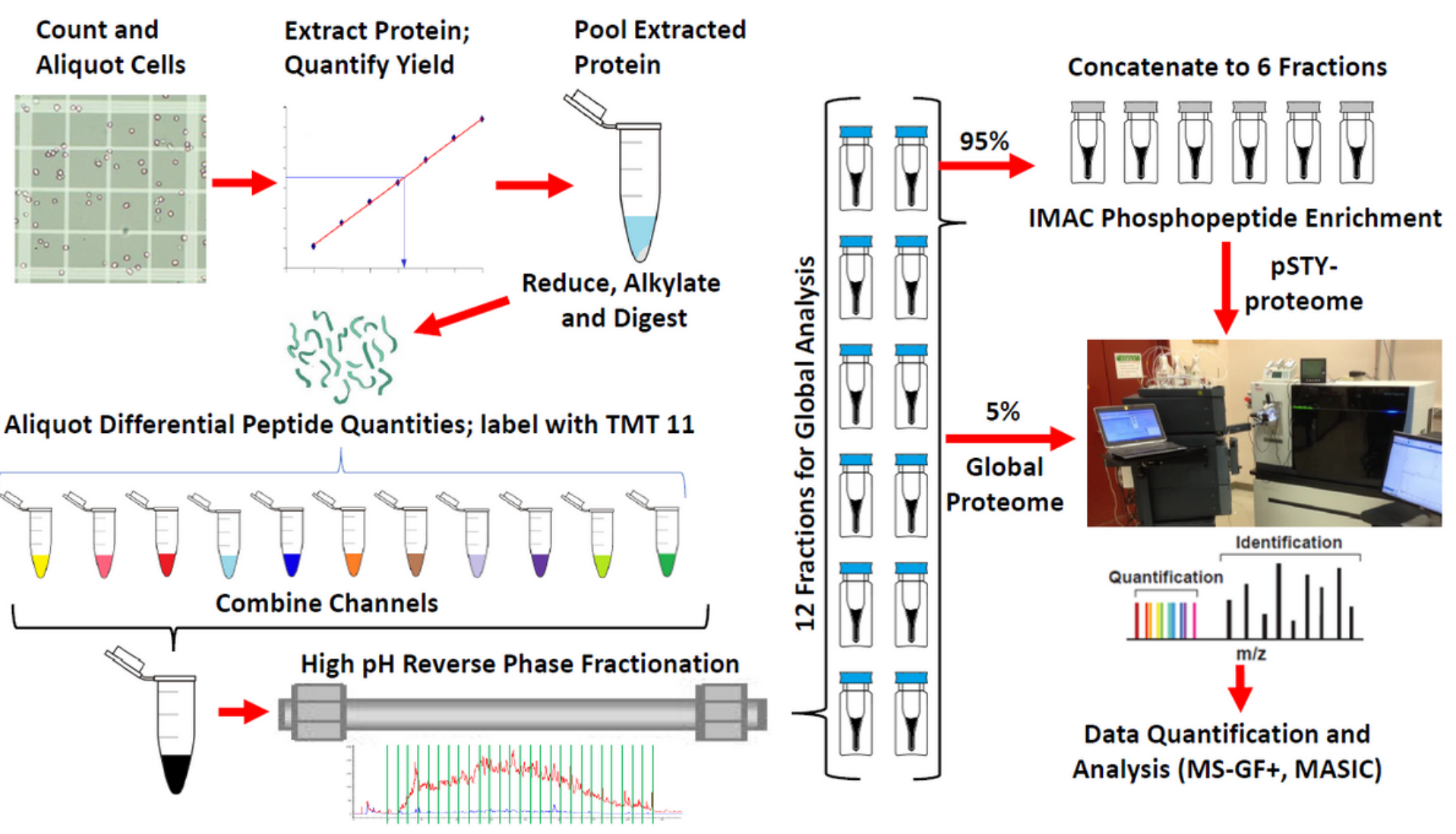

Figure 1

Clinical proteomics workflow. Diagram illustrating the steps involved in sample processing and data acquisition for our clinical proteomics workflow. 


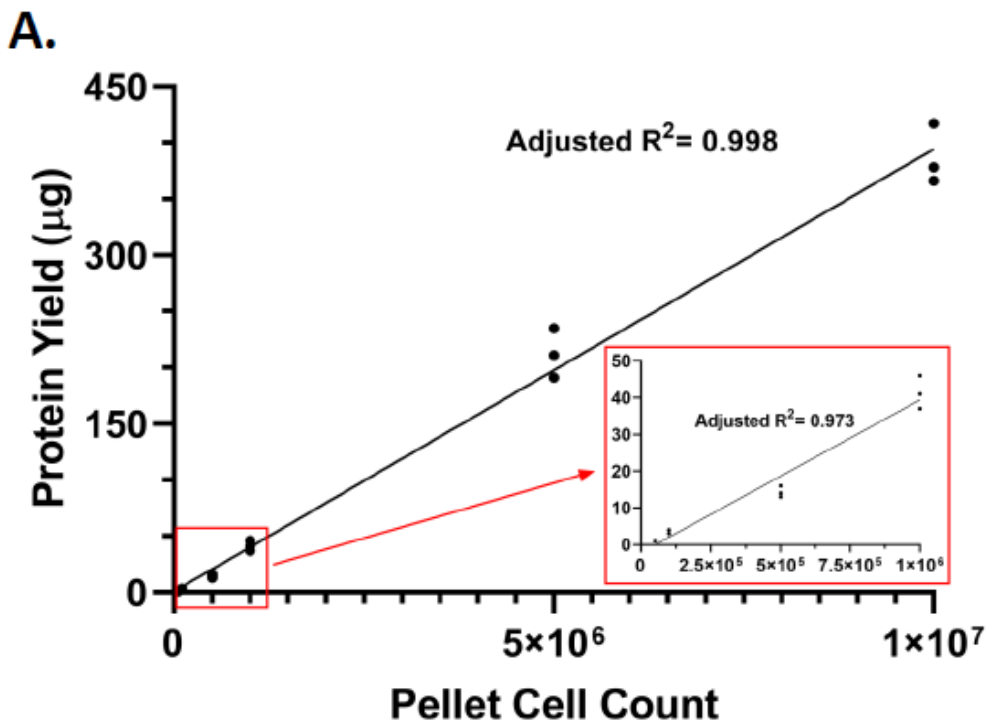

B.

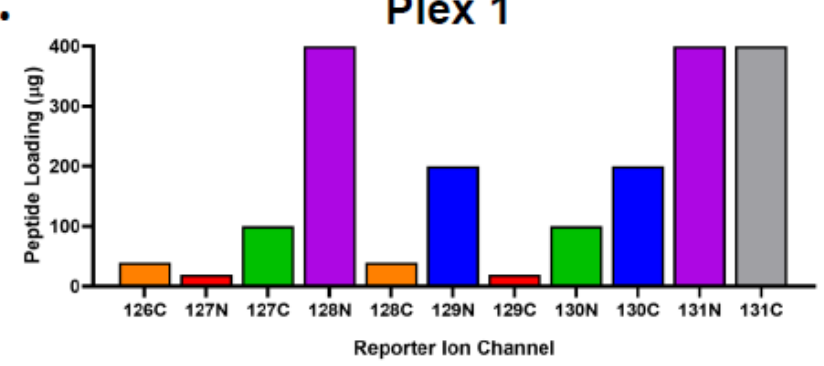

Plex 2

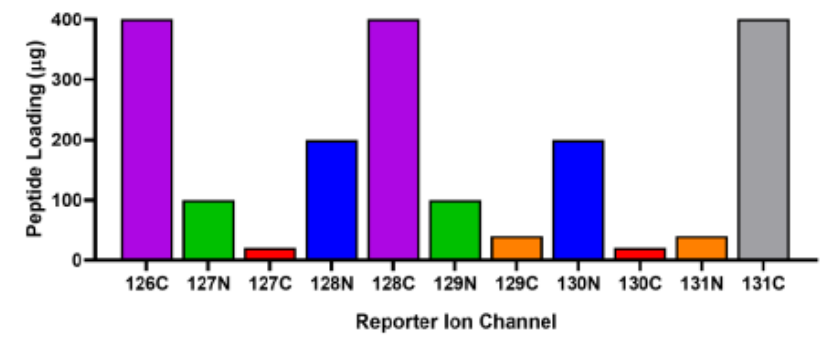

C. Plex 1

Labeling efficiency: $99.44 \%$

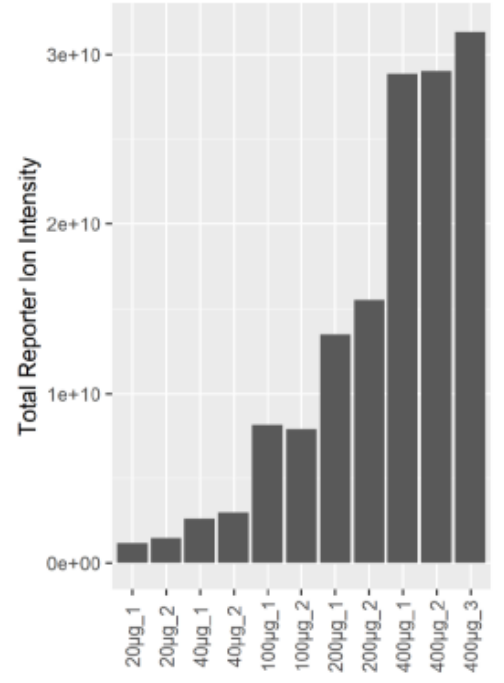

Plex 2

Labeling efficiency: $99.33 \%$

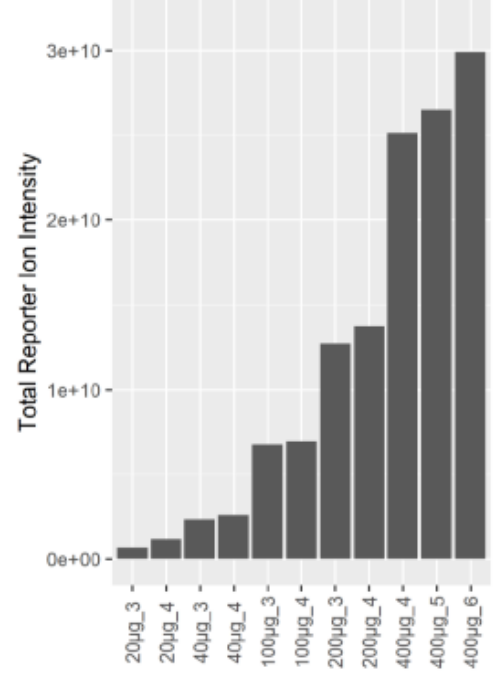

D.

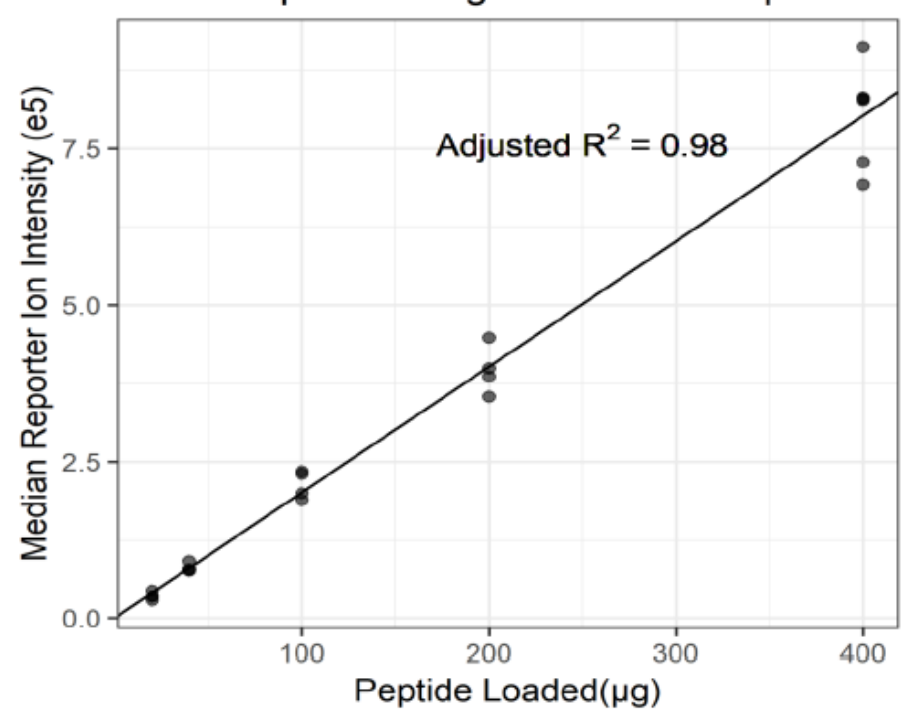

Figure 2

Design of TMT multiplexes with differential peptide loading. A) Protein extraction yields obtained from cell pellets of decreasing cell counts. B) Design of the two TMT11 multiplexes with differential amounts of peptide loaded per channel. C) Total TMT reporter ion intensity obtained per channel from global proteomics datasets. Additionally, TMT labeling efficiency was determined to be $>99 \%$ for each plex and is reported in the plot headers. D) Relationship between the amount of peptide loaded per channel and the median TMT reporter ion intensities acquired. 
A.

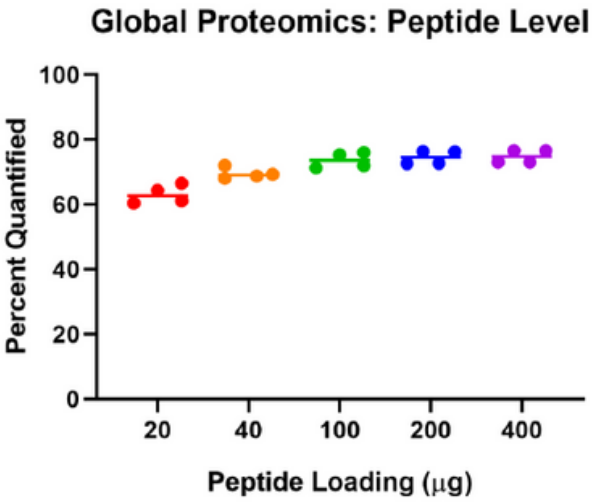

Global Proteomics: Protein Level

Phosphoproteomics: Peptide Level
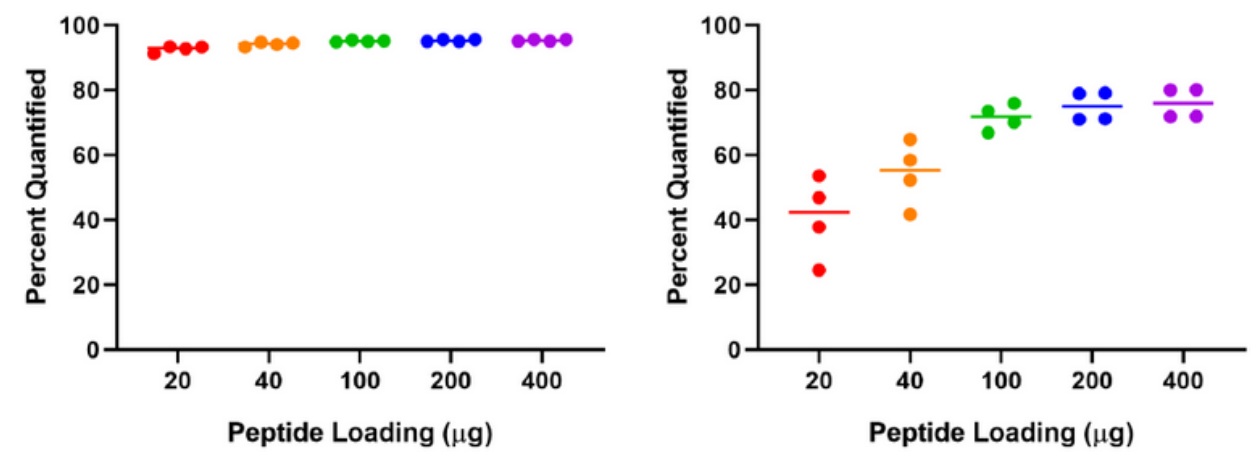

B.

Global, peptide level

C.

Phosphopeptide level
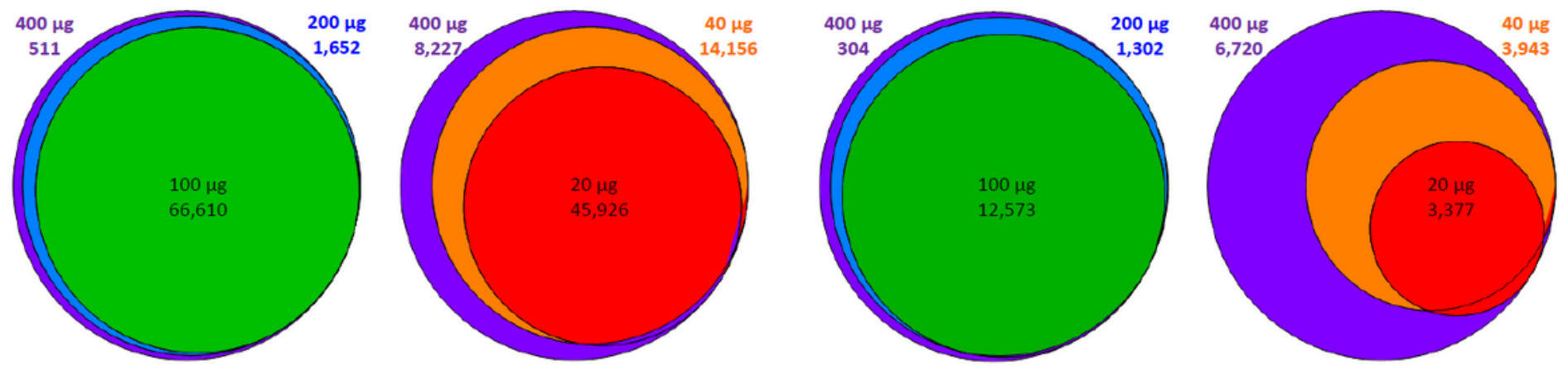

Figure 3

Missing data increases in channels with lower peptide loadings. A) Percentage of the total identified features (peptides or protein in global proteomics, phosphopeptides in phosphoproteomics) that are quantified in the 4 replicates of each peptide loading group. B-C) Venn diagrams demonstrating the overlap of peptides that were quantified (in all four replicates) of each differential peptide loading group. 
A. Global Proteomics
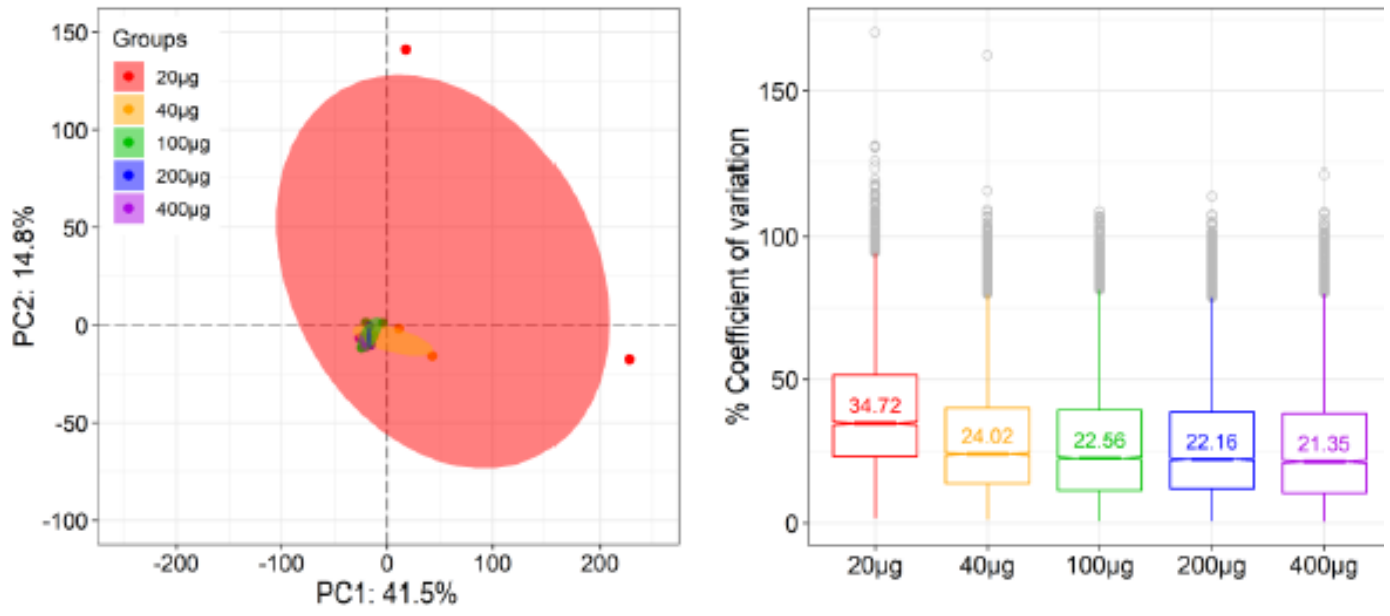

B.

Phosphoproteomics
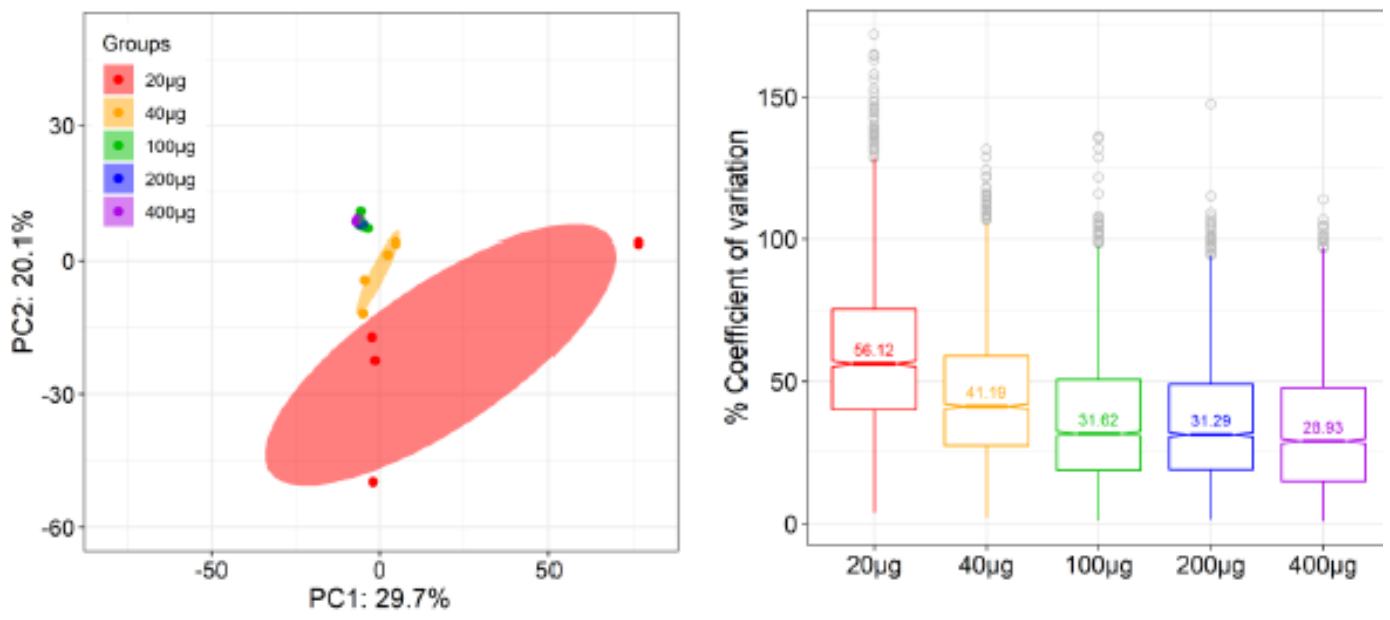

C. Global Protein Statistics

D. Phosphopeptide Statistics
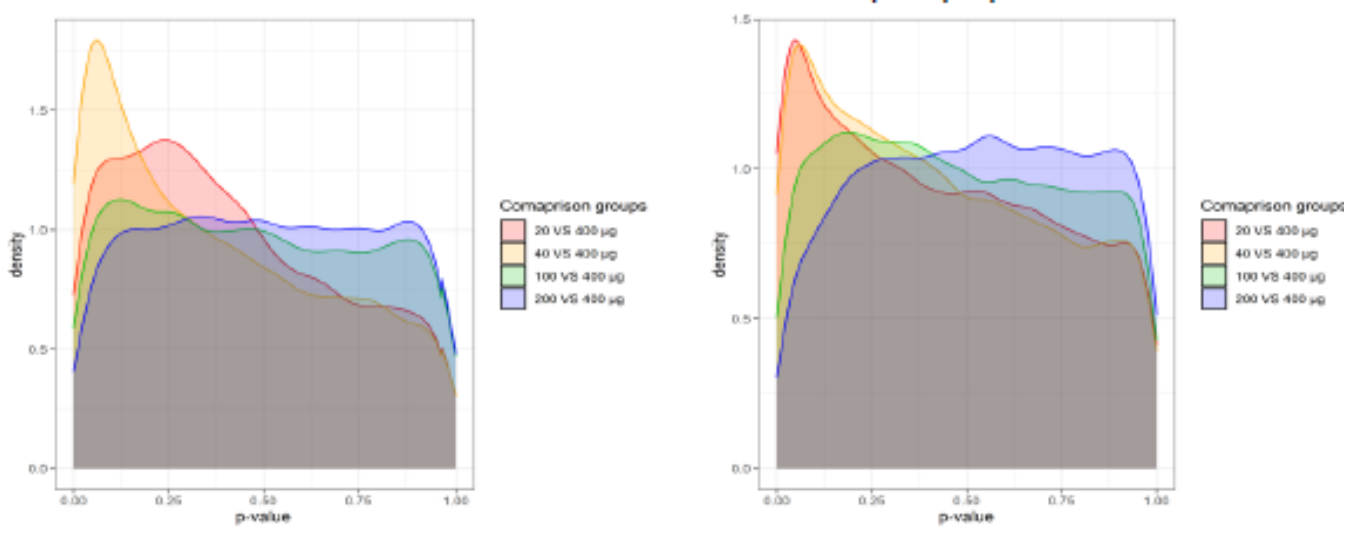

Figure 4

Impact of loading quantity on data reproducibility and statistics. A-B) Visualization of intra-group data reproducibility through principal component analysis (PCA) and coefficients of variation (\%CV) plots calculated across the four replicates of each loading group. C-D) Density plots of $p$-value histograms resulting from unequal variance t-tests comparing individual loading groups with the $400 \mu \mathrm{g}$ standard 
loading amount at the protein level from global proteomics datasets or peptide level from phosphoproteomics datasets.

\section{Supplementary Files}

This is a list of supplementary files associated with this preprint. Click to download.

- SuppFigure1.pdf

- SuppFigure2.pdf

- SupplementalTables.docx 